\title{
Spatial propagation of electrical signals in circular biofilms: A combined experimental and agent-based fire-diffuse-fire study
}

\author{
J. A. Blee $\odot,{ }^{1,2,3}$ I. S. Roberts, ${ }^{3, *}$ and T. A. Waigh ${ }^{1,2, \uparrow}$ \\ ${ }^{1}$ Biological Physics, School of Physics and Astronomy, University of Manchester, Oxford Road, Manchester, M13 9PL, United Kingdom \\ ${ }^{2}$ Photon Science Institute, Alan Turing Building, University of Manchester, Oxford Road, Manchester M13 9PL, United Kingdom \\ ${ }^{3}$ Lydia Becker Institute of Immunology and Inflammation Immunity \& Respiratory Medicine, Division of Infection, Immunity \& Respiratory \\ Medicine, School of Biological Sciences, Faculty of Biology, Medicine and Health, Manchester Academic Health Science Centre, Michael \\ Smith Building, University of Manchester, Oxford Road, Manchester, M13 9PT, United Kingdom
}

(Received 12 July 2019; revised manuscript received 13 September 2019; published 4 November 2019)

\begin{abstract}
Bacterial biofilms are a risk to human health, playing critical roles in persistent infections. Recent studies have observed electrical signaling in biofilms and thus biofilms represent a new class of active excitable matter in which cell division is the active process and the spiking of the individual bacterial cells is the excitable process. Electrophysiological models have predominantly been developed to describe eukaryotic systems, but we demonstrate their use in understanding bacterial biofilms. Our agent-based fire-diffuse-fire (ABFDF) model successfully simulates the propagation of both centrifugal (away from the center) and centripetal (toward the center) electrical signals through biofilms of Bacillus subtilis. Furthermore, the ABFDF model allows realistic spatial positioning of the bacteria in two dimensions to be included in the fire-diffuse-fire model and this is the crucial factor that improves agreement with experiments. The speed of propagation is not constant and depends on the radius of the propagating electrical wave front. Centripetal waves are observed to move faster than centrifugal waves, which is a curvature driven effect and is correctly captured by our simulations.
\end{abstract}

DOI: 10.1103/PhysRevE.100.052401

\section{INTRODUCTION}

Active excitable matter is extensively studied by both biological and nonlinear physics communities due to its primary role in human health, e.g., electrical waves across cardiac muscle [1-4]. It is challenging in that both the active rearrangement of the matter (e.g., growth of bacterial biofilms) and its excitability (e.g., spiking potentials of the bacteria) can contribute to emergent behavior.

Bacterial biofilms are communities of bacteria encased in a self-produced extracellular polymeric substance (EPS) [5-7]. Biofilms are responsible for most clinical infections [8] and related processes, such as biofouling, are costly to industry [9]. Central to this mode of growth is the ability of bacteria to coordinate behavior and act as a multicellular organism [5-7,10-12]. Therefore, understanding biofilm regulation is important in trying to control or prevent biofilm formation.

There are strong similarities between bacterial ion channels (with unknown roles) and eukaryotic ion channels involved in electrical signaling [13]. It is more difficult to study the electrophysiology of bacteria than eukaryotic cells $(\sim 10-100 \mu \mathrm{m})$ due to their small size $(\sim 0.2-2 \mu \mathrm{m})$. Recently, fluorescent probes were used by Prindle et al. [14] to provide the first direct evidence of electrical signaling between bacterial cells in a biofilm. Bacteria are also used in fuel cells to produce electricity [15], but there are again many fundamental gaps in our understanding.

\footnotetext{
*i.s.roberts@manchester.ac.uk

†ta.waigh@manchester.ac.uk
}

The Prindle study found that Bacillus subtilis cells within a two-dimensional biofilm (limited to a single cell thickness to facilitate imaging and modeling) communicate nutrient stress via electrical signaling. Inner biofilm cells responded to glutamate starvation by opening their potassium channels. This nutrient stress was then transferred to outer cells via a potassium wave. Outer biofilm cells then responded to this signal with periodic reductions in glutamate usage which allowed sufficient nutrients to reach the interior cells. The result of this coordinated behavior was an increase in the protection of the entire community from chemical attack. Further studies have shown that the role of electrical signaling in B. subtilis biofilms may extend beyond coordinating metabolic stress. The potassium signal was also shown to alter the motility of interacting planktonic B. subtilis and Pseudomonas aeruginosa cells [16]. Electrically conducting pili (nanowires) are also known to exist in a broad range of bacteria [17], although their role(s) predominantly remain unknown. This combined with the detection of voltage gated ion channels in the genome of a wide range of bacterial species [13] implies that electrical signaling may be a generic form of communication between bacteria.

The initiation and propagation of electrical signals in eukaryotic excitable tissues has been studied by electrophysiologists across a broad range of disciplines for well over a century [18]. Traditional biophysical methods (e.g., patch clamps, electrocardiograms, electroencephalography, etc.) combined with computer modeling have been central in this study and led to significant advances [19-21]. The study of bacterial electrical signaling is still only in its infancy. For a proper understanding and to allow comparison between different 
(a)

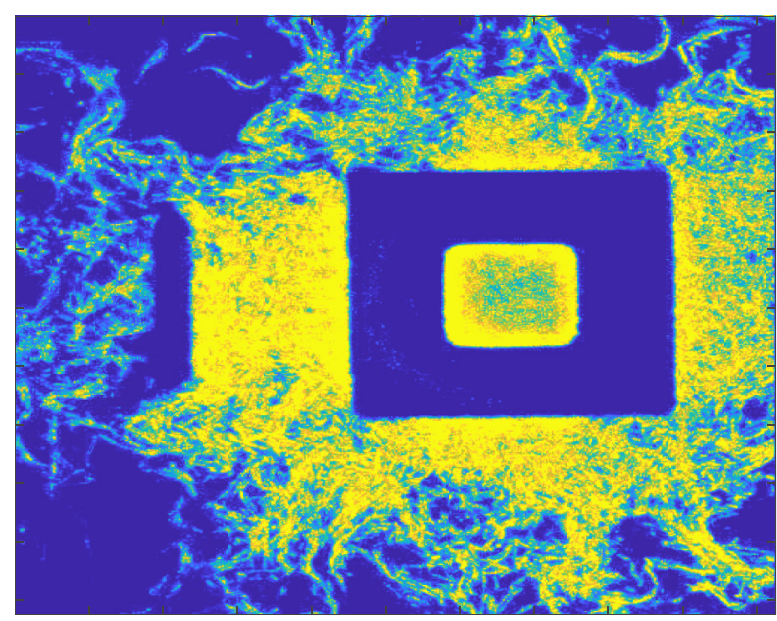

(b)

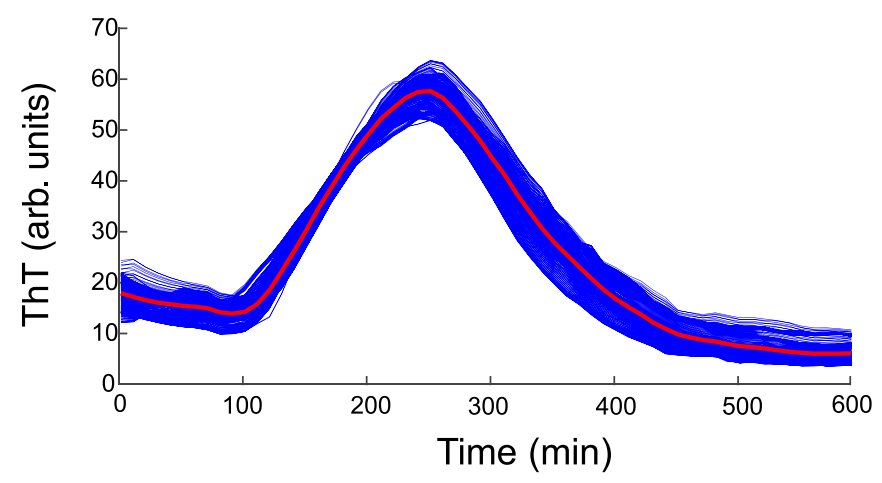

FIG. 1. Radially propagating electrical wave front in a biofilm. (a) A circular B. subtilis biofilm grown overnight in a microfluidic device (single cell thickness). Biofilm cells are stained with the membrane potential dye ThT. (b) ThT fluorescence observed at $4 \mu \mathrm{m}$ from the center of the biofilm shown in (a) as a function time. Signals from all angles are shown in blue and the average signal is shown in red.

biofilm signals, it is important that rigorous methods for the analysis and modeling of electrical signals in biofilms are established. Agent-based models are uniquely placed to offer insights into the behavior of complex systems, such as biofilms, due to their ability to integrate combinations of spatially heterogeneous processes [22-25]. Our study combines agent-based simulations with a fire-diffuse-fire (FDF) model. It provides a convenient extension to simple one-dimensional FDF models by describing the two-dimensional arrangement of bacteria in a biofilm and the emergent electrical wave fronts.

\section{MATERIALS AND METHODS}

\section{A. Cell preparation and biofilm growth}

All our experiments used the B. subtilis strain NCIB 3610. Biofilm growth was conducted following the protocols of Prindle [14]. The cells were freshly streaked onto lysogeny broth (LB) agar plates from glycerol stocks on the day before the experiment and incubated at $37^{\circ} \mathrm{C}$ overnight. The next day, $3 \mathrm{ml}$ of LB was inoculated with a single colony. The inoculum was then incubated and shaken at $200 \mathrm{rpm}$ at $37^{\circ} \mathrm{C}$ for approximately $3 \mathrm{~h}$ or until the cells reached an $\mathrm{OD}_{600}$ of $0.7-1.2$, at which stage the cells were centrifuged at $2100 \mathrm{rcf}$ for $1 \mathrm{~min}$ and then resuspended in a minimal MSgg medium to promote biofilm growth.

The MSgg medium was made from stock solutions on the day of the experiment as described by Branda et al. [26]. It contained: $5 \mathrm{mM}$ potassium phosphate buffer $(p \mathrm{H}$ 7.0), $100 \mathrm{mM}$ MOPS buffer ( $p \mathrm{H} 7.0$ adjusted using $\mathrm{NaOH}$ ), $2 \mathrm{mM} \mathrm{MgCl}_{2}, 700 \mu \mathrm{M} \mathrm{CaCl}_{2}, 50 \mu \mathrm{M} \mathrm{MnCl}_{2}, 100 \mu \mathrm{M} \mathrm{FeCl}_{3}$, $1 \mu \mathrm{MZnCl}_{2}, 2 \mu \mathrm{M}$ thiamine $\mathrm{HCl}, 0.5 \%$ (v/v) glycerol, and $0.5 \%(\mathrm{w} / \mathrm{v})$ monosodium glutamate.

Biofilms were grown in the CellASIC ONIX microfluidics system in Y04D chambers. Immediately after resuspension in MSgg the cell suspension was pipetted into the cell inlet well and the plate was sealed onto the microfluidic device. The chamber was then placed on the microscope which was incubated at $30^{\circ} \mathrm{C}$.

\section{B. Microscopy}

The Zeiss LSM 5 Pascal fluorescence microscope was used for phase contrast and fluorescence imaging. In general, a $20 \times$ objective lens was used to conduct time lapse studies and images were taken every $5 \mathrm{~min}$.

\section{Dyes}

Thioflavin-T (ThT) is commonly used to stain amyloid fibers; however, its positive charge also allows it to be used as a Nernstian voltage indicator. Its suitability in the role as a membrane potential indicator in bacterial cells was established by Prindle [14]. ThT was supplied by SigmaAldrich and it was used at $10 \mu M$. It has the advantage over other commonly used fluorescent voltage dyes of being more sensitive. For example, it was found in this setup by Prindle to have a threefold higher sensitivity to membrane potential changes than the commonly used membrane potential indicator $\operatorname{DiSC}_{3}(5)$.

\section{Data analysis}

Image analysis was conducted in MATLAB using custom made scripts. The $B$. subtilis cells grew in thin circular biofilms centered around flow traps [Fig. 1(a)] and this motivated the use of polar coordinates. Figure 1(b) is a representative ThT fluorescence profile as a function of time which shows the signals at all angles in a single radius and the average signal obtained by averaging these signals. The coefficient of variation of the signal's radial mean was always less than 0.07 . We therefore assumed that the average radial signal was representative of the whole radial signal. Background noise was subtracted and the offset signal due to the cell trap was negligible. Data were smoothed using a moving average filter. Graphs and fits were made in both MATLAB and ORIGIN. 
TABLE I. Parameters used for agent-based model of electrical signaling in a biofilm in GRO.

\begin{tabular}{lc}
\hline \hline Parameter & Value \\
\hline Simulation time conversion factor & $3.5 \mathrm{~min}$ \\
Simulation time step (dt) & $0.35 \mathrm{~min}$ \\
Pixel size & $0.1 \mu \mathrm{m}$ \\
Signal grid length & $20 \mathrm{pixel}(\mathrm{px})$ \\
Signal grid cell size & $20 \mathrm{px}^{2}$ \\
Cell growth rate & $0.034 \mathrm{fl} / \mathrm{min}$ \\
Average cell division size & $3.14 \pm 0.071 \mathrm{fl}$ \\
Potassium diffusion coefficient & $0.4($ molecules cell grid)/dt \\
Potassium degradation coefficient & 0.07 (molecules)/dt \\
\hline \hline
\end{tabular}

\section{E. Modeling}

Custom made MATLAB scripts were used to obtain solutions to the FDF model. To create our agent-based model, we used the extended version of GRO. Biofilm growth was controlled by CELLENGINE. CELLENGINE was developed to simulate large colonies and is optimized for rod shaped bacterium, such as $P$. aeruginosa, making it well suited for our purposes. During each simulation time step, each cell grows, which leads to an overlap. CELLENGINE resolves this overlap using rigidbody dynamics in two steps: collision detection and collision response. It does not support the growth of cells in chains and other more complex structures sometimes observed during biofilm growth.

The behavior of cells in GRO is controlled by a probabilistic timed automata-based library, CELLPRO, which encapsulates and simulates gene expression. In our model the release of potassium by a cell was implemented by a set of rules defined using CELLPRO. The propagation of potassium through the biofilm was implemented using CELLSIGNAL, which models signal propagation using a finite element model. At each time

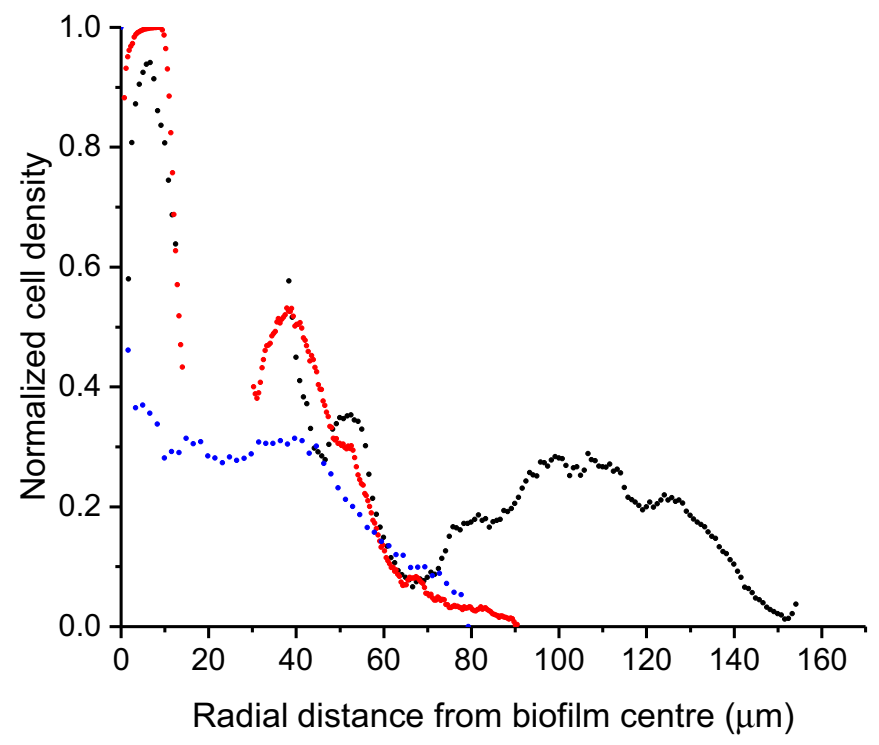

FIG. 2. Normalized cell density as a function of radial distance from the biofilm center for our experimental centrifugal wave front data (red), centripetal wave front data (black), and agent-based firediffuse-fire model (blue). The centripetal biofilm had a larger radius $(\sim 150 \mu \mathrm{m})$ than the centrifugal biofilm $(\sim 90 \mu \mathrm{m})$. step of the simulation, diffusion and degradation are applied to update the concentrations of the signals over a set of predefined grids. Further details regarding the algorithms used in CELLENGINE, CELLPRO, and CELLSIGNAL can be found in Gutiérrez et al. [27].

The GRO simulations were performed using experimentally relevant parameters. The units of GRO are currently not well defined. It is therefore necessary to associate units with the constants defined in a simulation. Table I shows the parameters used in our ABFDF model.

Figure 2 shows the cell density of our model which was chosen to match the experimental cell densities as closely as possible within the constraints of the assumptions implemented in GRO regarding cell growth and the cell interactions.

MATLAB was used to analyze the outputs of GRO and to create the potassium profile contour plots. ORIGIN was also used to create graphs and fits.

Custom made scripts for data analysis and modeling are publicly available [28].

\section{RESULTS}

\section{A. Propagation of electrical signals in biofilms (experimental results)}

Following the work of Prindle [14], we grew B. subtilis biofilms under flow in the CellASIC ONIX microfluidic system and monitored the membrane potential using the membrane potential indicator Thioflavin-T (ThT). The higher the ThT concentration in the bacteria, the lower their membrane potential.

In our experiments both outward moving [centrifugal, Fig. 3(a)] and inward moving [centripetal, Fig. 3(b)] waves were observed. Figure 3(c) shows the electrical signals observed at different radii for a typical centrifugal electrical wave traveling outward through a biofilm. To quantify these electrical signals Prindle [14] defined their half maximal position (in time) and their amplitude. However, they can be more accurately described using moments. Moments analysis provides an alternative method to describe probability distributions [29]. We considered standard parameters based on the first four moments of the distributions, i.e., the mean, the standard deviation, the kurtosis, and the skewness, Eqs. (1)-(4), since they could be robustly calculated from our experimental data and have relatively simple intuitive interpretations. The skewness is a measure of the distribution's symmetry and the kurtosis is an indicator of whether it is peaked and has a heavy tail.

The $n$th moment of a distribution $f(x)$ with $N$ points is given by

$$
\left\langle x^{n}\right\rangle=\sum_{i=1}^{N} x_{i}^{n} f(x) .
$$

The mean $(\mu \equiv E[X])$ of the distribution is given by the first moment. The standard deviation is given by the square root of the variance, which is given by the second central moment,

$$
\left.\sigma=\sqrt{E\left[(x-\mu)^{2}\right.}\right] \text {. }
$$

The third and fourth moments of a distribution can be used to calculate the skewness and the kurtosis. The skewness is 


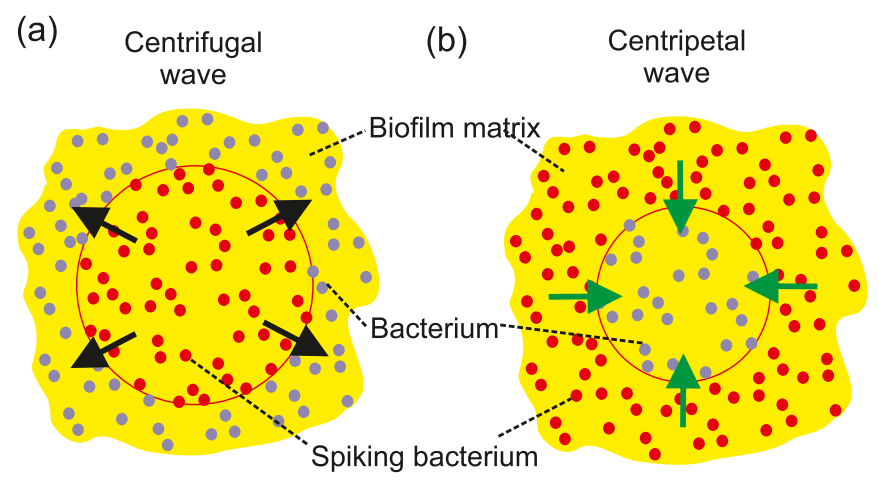

(c)

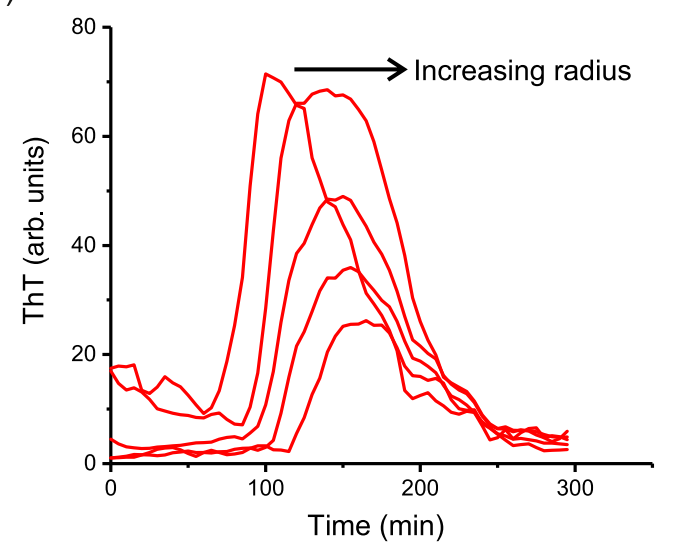

FIG. 3. Electrical signal propagation through a two-dimensional biofilm. Schematics show the spread of (a) centrifugal ("away from the center") and (b) centripetal ("toward the center") electrical wave fronts through a biofilm. (c) Electrical signal indicated by ThT fluorescence as a function of time at five different biofilm radii $(r=2,10,15,100$, and $150 \mu \mathrm{m})$ from fluorescence microscopy experiments.

defined as

$$
S=E\left[\left(\frac{X-\mu}{\sigma}\right)^{3}\right],
$$

and the kurtosis is defined as

$$
K=E\left[\left(\frac{X-\mu}{\sigma}\right)^{4}\right] .
$$

Prindle originally only described electrical wave fronts which originate at the center of the biofilm (centrifugal wave fronts [Fig 3(a)]). In addition, we experimentally observed electrical wave fronts which originate at the outer biofilm (centripetal wave fronts [Fig 3(b)]). Figure 4 shows both the membrane potential profile as a function of time observed experimentally for a centrifugal wave front [Fig. 4(a)] and a centripetal wave front [Fig. 4(b)]. Biofilms grown in the microfluidics system grew out from a cell trap, which led to the small gaps in the data seen in Fig. 4. The fluorescent energy density $\left[E_{r}(r)\right]$ of both centrifugal and centripetal waves decreased sigmodially with radial distance from the biofilm center,

$$
E_{r}(r)=\frac{1}{1+e^{\left(r-r_{0}\right) / x}},
$$

(a)

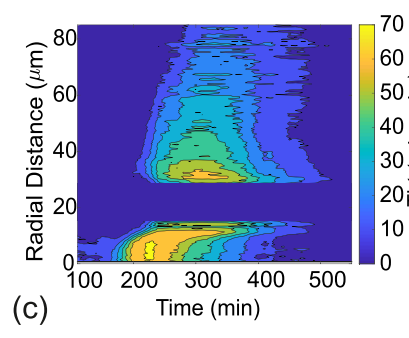

(b)
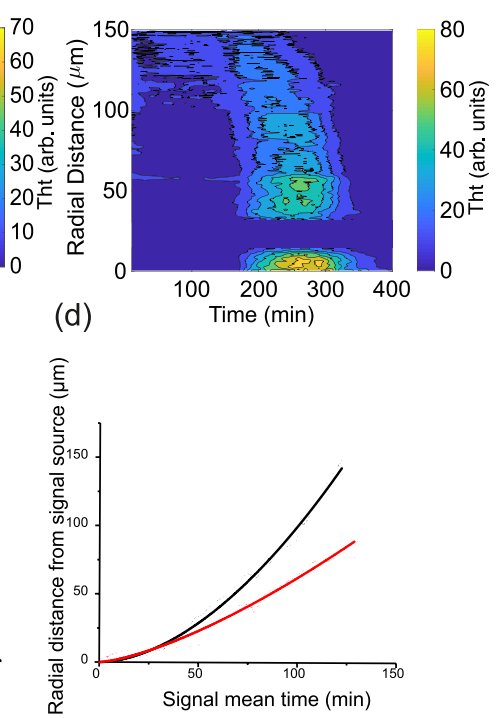

FIG. 4. Propagation of centrifugal and centripetal electrical signals through a biofilm. (a,b) ThT fluorescent intensity as a function of time and radial distance for a biofilm in which an electrical signal has (a) originated from the biofilm center (centrifugal) and (b) from the biofilm edge (centripetal). (c) The signals' fluorescence energy density as a function of radial distance for the centrifugal wave front (red) shown in (a) and for the centripetal wave front (black) shown in (b), fitted with sigmoids, Eq. (5). (d) Radial distance for the maximum intensity as a function of signal mean time for the centrifugal wave front (red) shown in (a) and the centripetal wave front (black) shown in (b), fitted with power laws, Eq. (7).

where $r_{0}$ is the half radial constant and $x$ is the slope constant, which describes the steepness of the curve.

The average skewness of the centrifugal wave front was $-0.03 \pm 0.18$ and the centripetal skewness was $-0.32 \pm 0.15$. The skewness of the centrifugal wave decreased as it traveled, whereas the skewness of the centripetal wave increased as it traveled [Fig. 9(a)].

The centrifugal kurtosis was $3.78 \pm 0.28$ and the centripetal kurtosis was $2.29 \pm 0.17$. The kurtosis of the centrifugal wave was greater than 3, (leptokurtic, more peaked than a Gaussian), whereas the centripetal kurtosis was less than 3 (platykurtic, less peaked than a Gaussian).

The kurtosis values indicated that the signals were not perfect Gaussians. However, after the data were smoothed using moving averages, Kolmogorov-Smirnov tests were performed at the 5\% significance level to confirm that the data were well approximated by Gaussians of the form

$$
F_{r}(t)=\frac{a_{r} e^{-\left(t-b_{r}\right)^{2}}}{c_{r}}
$$

where $F_{r}(t)$ is the average ThT fluorescence as a function of time at the radius $r, a_{r}$ is the average peak fluorescence of the signal at radius $r, b_{r}$ is the average time of the peak amplitude of the signal at $r$, and $c_{r}$ is a constant related to the standard deviation giving the width of the signal at $r$.

Gaussian fits were therefore used to robustly obtain the radial signal amplitude. As expected, the signal amplitude followed the same profile as the fluorescence energy 
density (found directly using radial averages of images) and decreased sigmoidally with radial distance from the biofilm center Eq. (5).

Figure 4(d) shows the propagation of the maximum of the signal through the biofilm (velocity profiles of the maximum). These velocity profiles followed a power law dependence of distance on time,

$$
D=A_{0} \tau^{\alpha},
$$

where $D$ is the radial distance the signal has traveled; $\tau$ is the mean time $(\mu)$, which is the time at which the signal maximum is reached at a specific $D$; and $A_{0}$ and $\alpha$ are both constants.

The centripetal wave had a steeper profile than the centrifugal wave, with an exponent $(\alpha)$ of $1.79 \pm 0.03$ rather than $1.42 \pm 0.06$. This suggests that it traveled faster. It is well established that curvature can affect the propagation of a wave front through an excitable medium [30-32]. For a centripetal wave front, different parts of the front will excite the same point concentrating its activity, whereas for a centrifugal wave front, which expands as it travels, the energy to excite neighbors is spread out. It was therefore expected that, if these waves occurred via the same mechanism, the centrifugal wave should travel slower than the centripetal wave, as was observed. For both wave fronts (centripetal and centrifugal) the exponent was significantly larger than $1(\alpha>1)$. This shows that the wave fronts were not propagated constantly as previously described by Prindle [14].

\section{B. Modeling electrical signaling in biofilms \\ 1. Fire-diffuse-fire model}

Fire-diffuse-fire (FDF) models were originally developed to describe intracellular calcium propagation [33]. We tested whether potassium propagation in a biofilm may be described by a similar model. In this model once a threshold concentration of potassium $\left(k^{*}\right)$ is reached at a single cell, the cell fires instantaneously releasing a fixed concentration of potassium $(\sigma)$. A potassium wave was therefore propagated by the sequential firing of biofilm cells. In this FDF model in one dimension the potassium profile $[K=K(x, t)$, where $x$ is the position and $t$ is the time] obeys the reaction-diffusion equation,

$$
\frac{\partial K}{\partial t}=D_{k} \frac{\partial^{2} K}{\partial x^{2}}-\gamma K+\sigma \sum_{i} \delta(x-i L) \delta\left(t-t_{i}\right),
$$

where $D_{k}$ is the potassium biofilm diffusion coefficient, $i L$ is the location of the $i$ th cell, $t_{i}$ is the time when the threshold value of potassium $\left(k^{*}\right)$ is reached at the $i$ th cell, and $\gamma$ is the potassium decay rate. This reaction-diffusion equation has an exact solution in one dimension given by

$$
\begin{aligned}
K(x, t) & =\sum_{i} K_{i}(x, t), \\
K_{i}(x, t) & =\frac{\sigma H\left(t-t_{i}\right)}{\sqrt{4 \pi D\left(t-t_{i}\right)}} \exp \left[-\frac{(x-i L)^{2}}{4 D\left(t-t_{i}\right)}-\gamma\left(t-t_{i}\right)\right],
\end{aligned}
$$

where $K_{i}(x, t)$ is the potassium profile of a single cell and $H$ is the Heaviside function. For a wave to propagate steadily, (a)

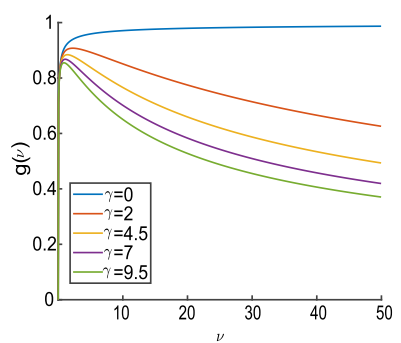

(c)

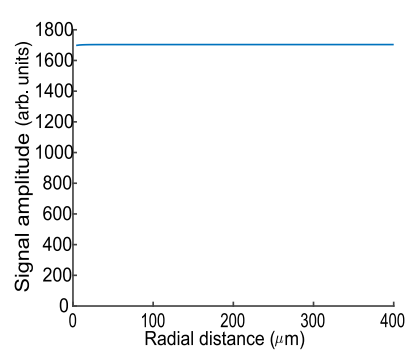

(b)

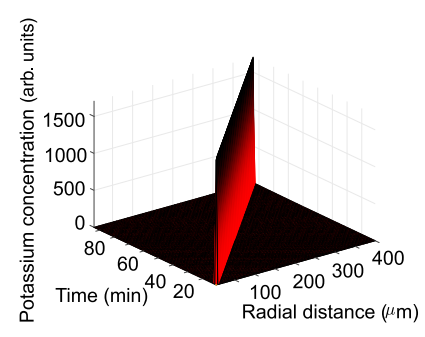

彥 $(d)$

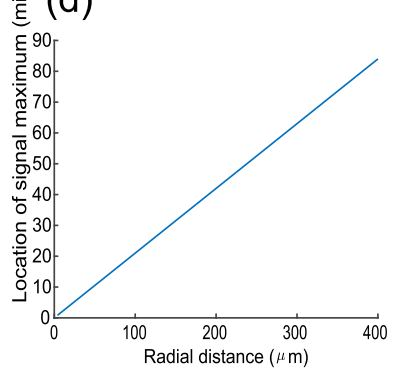

FIG. 5. Fire-diffuse-fire model of electrical signal propagation through a biofilm. (a) Plot of $g(v)$ as a function of $v$ for a range of different $\gamma \cdot g(v)$ is a function which may be used to determine the model's stability and thus find constantly propagating solutions to the FDF model. (b) Potassium signal produced by our FDF model of a biofilm, Eq. (9). (c) Signal amplitude of the potassium wave shown in (b). (d) Velocity profile (position of the signal maximum as a function of time) of signal shown in (b).

the time between firing of consecutive cells must be constant $\left(t_{i}-t_{i-1}=\tau\right)$.

To model the results of Prindle [14], we first took the same propagation timescale $(\tau)$ as $0.84 \mathrm{~s}$, assumed a cell separation $(L)$ of $1 \mu \mathrm{m}$, and that the potassium diffusion coefficient in the biofilm was $70 \%$ of its aqueous value $\left(D_{k}\right.$ is $\left.1380 \mu \mathrm{m} \mathrm{s}^{-1}\right)$ [34].

For a wave to propagate steadily the time between firing of consecutive cells must be constant $\left(t_{i}-t_{i-1}=\tau\right)$ for all cells. If such a $\tau$ exists, then it is a solution of the equation [33]

$$
\frac{k^{*} L}{\sigma}=\sum_{n=1}^{\infty} \frac{1}{\sqrt{4 \pi n v}} \exp \left(\frac{-n}{4 v}-\beta^{2} n\right) \equiv g(v),
$$

where $v=\frac{D \tau}{L^{2}}$ and $\beta^{2}=\frac{\gamma L^{2}}{D}$.

In order to determine if constantly propagating solutions exist, we plotted $g(v)$ for a range of different experimentally relevant parameters, for varying values of $\gamma$ [Fig. 5(a)]. If $\frac{k^{*} L}{\sigma}>g(v)_{\max }$ propagation failed, we were therefore only interested in solutions $0<g(v)<g(v)_{\max }$. Figure 5(a) shows that these solutions existed for all the tested $\gamma$ values, proving that the FDF model may be used to obtain a constantly propagating electrical signal in the biofilm within experimentally relevant parameters.

Figure 5(b) shows profiles produced by our FDF model using $\tau=0.84 \mathrm{~s}, L=1 \mu \mathrm{m}, D_{k}=1380 \mu \mathrm{m} / \mathrm{s}$, and $\beta=0.05$. Figures 5(c) and 5(d) show the amplitude and propagation of this signal through the biofilm, respectively.

A simple FDF model can therefore be used to model an electrical signal that propagates constantly through a biofilm. 
This FDF model is a simple 1D model and it is also limited by the assumptions that the biofilm cells' positions are modeled as delta functions and firing is instantaneous. It is, however, useful due to its simplicity and its exact solution. We suggest that such a model may be useful for studying propagation failure or success in biofilms (i.e., how stable electrical signaling waves can be sustained). It is not, however, useful for interpreting our experimental results, since realistic analytical solutions for a wave front which does not propagate constantly do not exist for Eq. (8) in two dimensions.

\section{Agent-based model}

The propagation of an electrical signal through a biofilm does not always follow a constant velocity and amplitude as originally proposed by Prindle [14] (Fig. 3). To understand more complicated patterns and accurately describe biofilms in two dimensions, we turned to agent-based modeling (ABM) [22]. ABMs allow complex global behaviors to be described which emerge as a result of interactions between individuals in a multicellular community, e.g., accurate placement of bacteria in a $2 \mathrm{D}$ biofilm with varying density. In our ABM each cell in the biofilm was modeled as an "agent" which obeyed simple rules.

A range of different software packages have been developed to simulate cell colonies. We chose to build our ABM using the extended version of GRO [27] that functions well in two dimensions (2D), because it is a simple and fast simulator, which has been optimized for understanding the effects of colony spatial arrangement on cell-cell communication. The extended version of GRO relies on five major components:

(1) A CELLENGine is a physics engine which was developed to simulate large colonies and is optimized for rod shaped bacterium, such as B. subtilis, making it well suited for our purposes.

(2) PROSPEC is an extension to CCL [35], a guarded command-based language developed for modeling cooperative systems.

(3) CELLPRO is a probabilistic timed automata-based library that simulates gene expression dynamics using digital proteins. These proteins are then used to drive cell behavior.

(4) The additional libraries CELLNUTRIENT and CELLSIGNALS can be used in addition to control the external environment. Intercellular and environmental signaling is implemented through a set of grids that store the signal concentration at each grid location. At each time step of the simulation, diffusion and degradation are applied to update the concentrations of the signals over the whole grid using a finite element model and the cell states are then updated.

(5) GRO is the central subsystem of the simulator.

Figure 6 shows the workflow executed for each time step of our simulations inside GRO.

We built an agent-based model based on the FDF model (ABFDF model) using GRO. Each bacterial cell in the biofilm is considered as an agent. In the simulations potassium release was triggered either at the central cell (centrifugal) or at an edge ring of cells (centripetal). This potassium wave was then actively propagated via the triggering of potassium release at other cells. Following the FDF model, potassium release was triggered at a cell after a threshold concentration of potassium

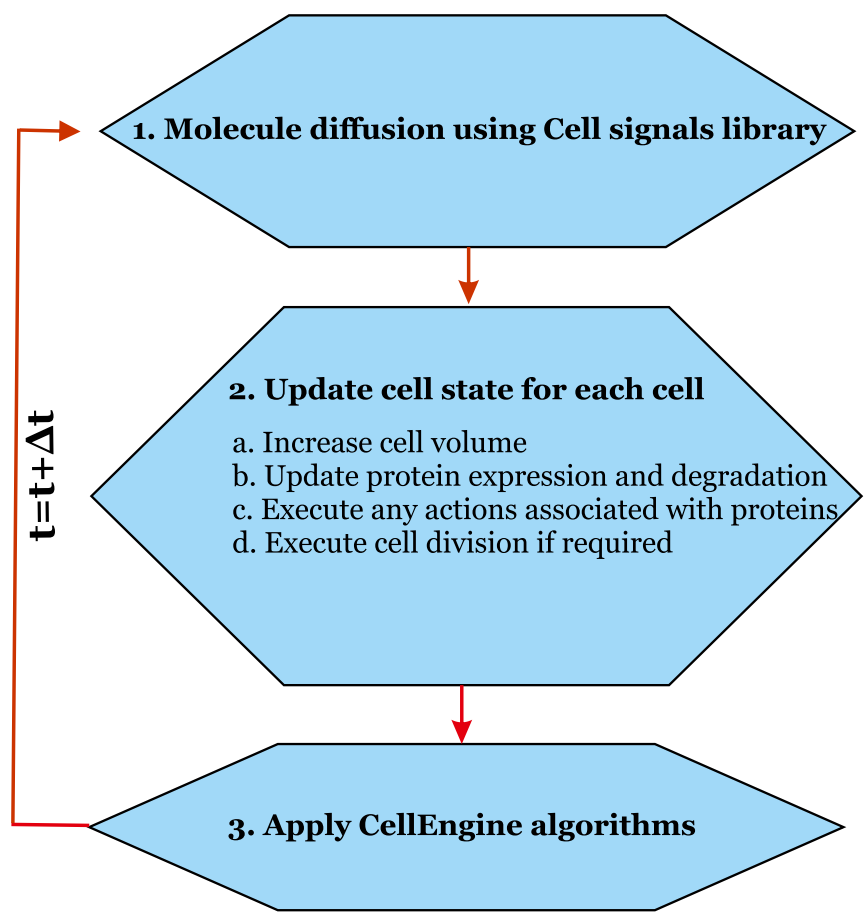

FIG. 6. Workflow showing the steps executed by our model per time step $(\Delta t)$.

was reached at that cell. The release function of a single cell $\left(k(t)_{j}\right)$ was described by a rectangular function,

$$
k(t)_{j}= \begin{cases}0 & \text { if } t<t_{j} \\ \varepsilon & \text { if } t_{j} \leqslant t \leqslant t_{j}+t_{r}, \\ 0 & \text { if } t>t_{j}+t_{r}\end{cases}
$$

where $\varepsilon$ is the amplitude of the potassium concentration; $t_{j}$ is the time of release at cell $j$; and $t_{r}$ is the rise time, which is how long each cell fires potassium.

Figure 7 shows a GRO simulation of this ABFDF model. The bacterial density profile of the simulated biofilm (i.e., the areal concentration as a function of radial distance) was matched with the experimental data (Fig. 2) and this was found to be crucial to accurately model the propagation of electrical waves. Figure 8 shows the potassium profile produced by our GRO simulation for (a) a centrifugal wave front and (b) a centripetal wave front. The fluorescent energy density and amplitude of the signals [Fig. 8(c)] decreased sigmoidally with radial distance from the biofilm center as seen for our experimental data [Fig. 4(c)] for both centripetal and centrifugal waves. However, as reflected by the constants defining these sigmoids, the amplitude of the signals produced by our model did not decrease as rapidly with radial distance as was observed experimentally (Table II).

The average kurtosis of the model centrifugal signal is $3.812 \pm 0.004$ which matches our experimental results (3.78 \pm 0.28 ). The average kurtosis of the centripetal model signal is $3.21 \pm 0.01$, which, in agreement with our experimental results $(2.29 \pm 0.17)$, is lower than the average kurtosis of the centrifugal signal, but was still larger than our experimental results. The average skewness of the centrifugal model wave front was $0.548 \pm 0.004$ and the model centripetal wave front 
(a)

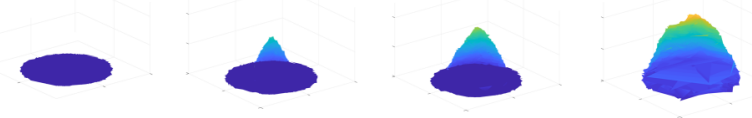

(b)

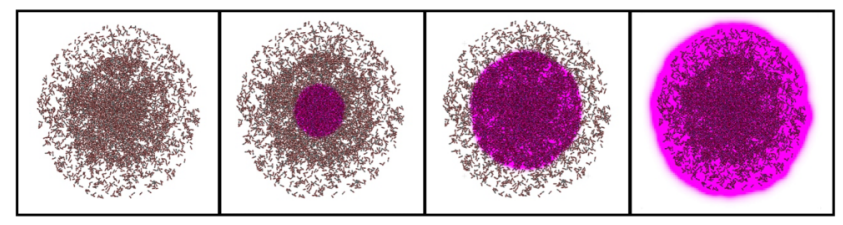

(c)

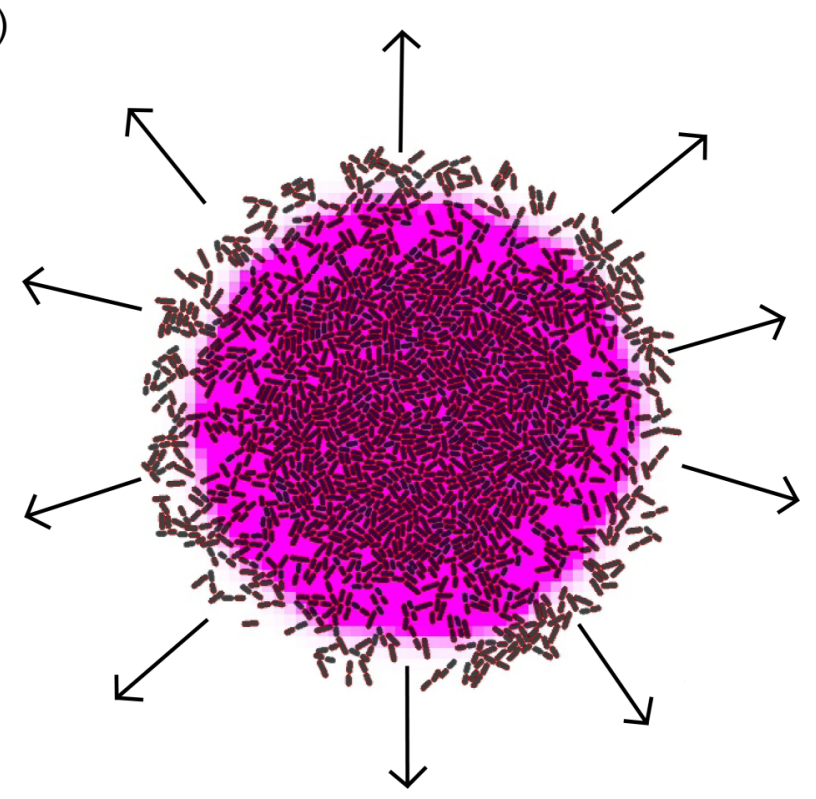

FIG. 7. Time-lapse images from a simulation using the agentbased fire-diffuse-fire model for two-dimensional circularly symmetric biofilms shown in (a) three dimensions and (b) two dimensions. Snapshots are shown for time since initial firing at the center of the biofilm; $T=0,17,63$, and $120 \mathrm{~min}$. (c) A magnified image of a potassium wave spreading out from the center of the biofilm simulated by our agent-based fire-diffuse-fire model where the bacterial agents are clearly visible.

had an average skewness of $0.719 \pm 0.001$. This indicates that the wave fronts produced by our model had tails to the right in contradiction with our experimental results.

Closer examination of the changes in skewness observed with propagation through the biofilm indicated that these (a)

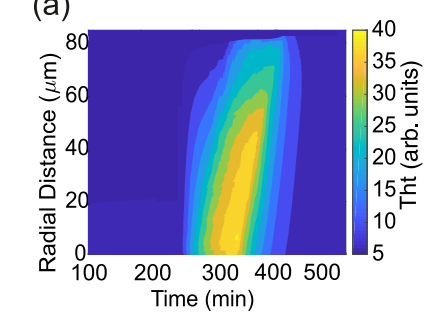

(c)

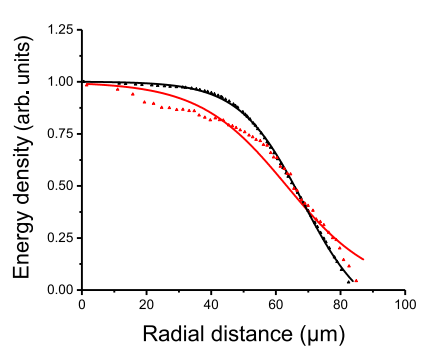

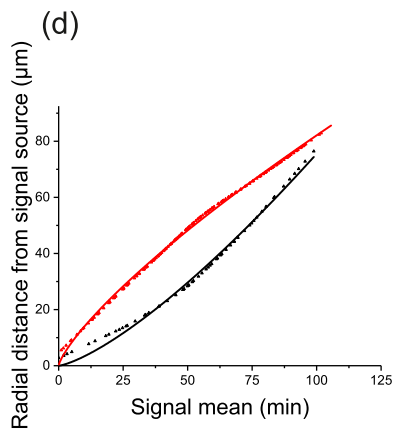

(b)

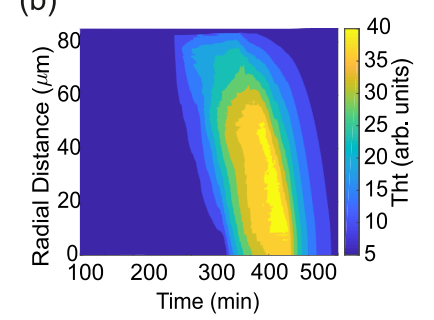

FIG. 8. Propagation of (a) centripetal and (b) centrifugal electrical waves produced by our agent-based fire-diffuse-fire model. The potassium profiles were produced by our model for a signal triggered at (a) the biofilm center and (b) the biofilm edge. (c) Fluorescence energy density, as a function of radial distance, of the centripetal signal (red) and for the centrifugal signal (black) fitted with sigmoids, Eq. (5). (d) Radial distance for the maximum intensity as a function of signal mean time for the centripetal signal (red) shown in (a) and the centrifugal signal (black) shown in (b), fitted with power laws, Eq. (7). For (c), (d) data were averaged over three separate simulations.

differences may be explained by differences in biofilm size and the presence of the cell trap in the microfluidic flow cell. Figure 9 shows the kurtosis and skewness of the experimental results (a) and (b), as well as our model results (c) and (d). Curves shown in red are centrifugal and black are centripetal. This Figure shows that the underlying trends in the model and experimental skewness and kurtosis were more comparable than the averages suggest that the underlying trends between the centrifugal and centripetal wave front are more comparable than the averages may suggest. The skewness of the centrifugal wave front was larger than the centripetal wave front, with a decrease in the skewness observed with the direction of the wave front. The kurtosis of the centrifugal wave front was also larger than the centripetal wave front.

The velocity profiles were fitted with power laws [Eq. (7), Table II]. The profile of the simulated centripetal wave front was steeper than the simulated centrifugal wave front

TABLE II. Fit constants of Eqs. (5) and (7) for our experimental and model wave fronts.

\begin{tabular}{lccccc}
\hline \hline $\begin{array}{l}\text { Fit } \\
\text { constant } \\
\text { name }\end{array}$ & $\begin{array}{c}\text { Fit } \\
\text { constant } \\
\text { symbol }\end{array}$ & $\begin{array}{c}\text { Experimental } \\
\text { centrifugal } \\
\text { wave front }\end{array}$ & $\begin{array}{c}\text { Experimental } \\
\text { centripetal } \\
\text { wave front }\end{array}$ & $\begin{array}{c}\text { Model } \\
\text { centrifugal } \\
\text { wave front }\end{array}$ & $\begin{array}{c}\text { Model } \\
\text { centripetal } \\
\text { wave front }\end{array}$ \\
\hline $\begin{array}{l}\text { Fluorescent energy densities half radial constant (arb. units) } \\
\text { Fluorescent energy densities slope constant (arb. units) }\end{array}$ & $r_{0}$ & $41.1 \pm 0.5$ & $44.7 \pm 0.4$ & $63.4 \pm 0.1$ & $68.4 \pm 0.1$ \\
Power law constant for wave velocity profile & $x$ & $7.5 \pm 0.5$ & $24.2 \pm 0.4$ & $13.5 \pm 0.1$ & $9.8 \pm 0.1$ \\
Exponent constant for wave velocity profile & $\alpha$ & $0.081 \pm 0.021$ & $0.026 \pm 0.003$ & $2.441 \pm 0.001$ & $0.131 \pm 0.006$ \\
$A_{0}$ & $1.42 \pm 0.06$ & $1.79 \pm 0.03$ & $0.76 \pm 0.01$ & $1.38 \pm 0.01$ \\
\hline \hline
\end{tabular}


(a)

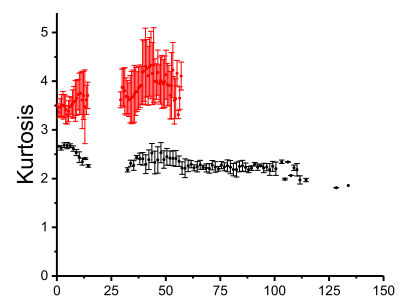

Radial distance from biofilm centre $(\mu \mathrm{m})$

(c)

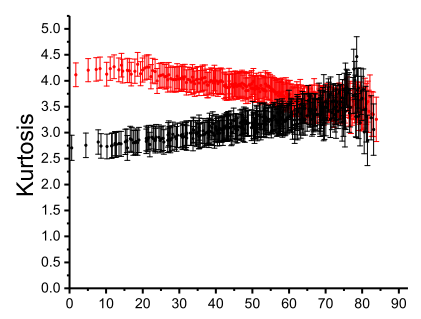

Radial distance from biofilm centre $(\mu \mathrm{m})$ Radial distance from biofilm centre $(\mu \mathrm{m})$

FIG. 9. Kurtosis and skewness of the electrical signal as a function of radial distance. (a) Kurtosis of our experimental centrifugal wave front (red) and centripetal wave front (black). (b) Skewness of our experimental centrifugal wave front (red) and centripetal wave front (black). (c) Kurtosis of our ABFDF model's centrifugal wave front (red) and centripetal wave front (black). (d) Skewness of our ABFDF model's centrifugal wave front (red) and centripetal wave front (black).

[Fig. 8(d)] in agreement with our experimental results, as well as the theory that the curvature affects the propagation speed [30-32]. However, our model was not fully successful in producing the experimental velocity characteristics. The exponents of the power laws fitted to our model results were lower than was observed experimentally. The simulated centripetal wave front had an exponent of $\alpha=1.38 \pm 0.01$, whereas the experimental exponent was $\alpha=1.79 \pm 0.03$ (Table II). The simulated centrifugal wave front had an exponent of $\alpha=0.76 \pm 0.01$, whereas the experimental wave front had an exponent of $\alpha=1.42 \pm 0.06$. It is likely that these differences were caused by the complicated experimental set-up, as well as by the models assumption that cells did not grow in chains or other complex geometrical arrangements often observed during biofilm growth. During our experiments outer biofilm cells clustered and formed chains, which was not accounted for in our model.

In summary, the spatial arrangement of cells (the curvature of the biofilm and variations in cell density) can explain the majority of the differences in the propagation of the centrifugal and centripetal electrical wave fronts. However other, more subtle, propagation characteristics (e.g., fractional power law velocity profiles) were not quantitatively described by our model owing to the complex nature of biofilm growth.

\section{DISCUSSION AND CONCLUSION}

Following the work of Prindle [14] we studied B. subtilis biofilms and found that the electrical signals did not propagate constantly through the biofilm. We also found that, in addition to the originally described centrifugal wave fronts, there were wave fronts which originated at the biofilm edge, i.e., centripetal wave fronts. The fluorescence energy density and amplitude of both wave fronts decreased with distance from the biofilm center, regardless of the signal's origin. The shape of the signal, which was characterized by the skewness and kurtosis, was dependent on the direction of travel. As previously described for other excitable systems [30-32], the centrifugal wave traveled slower than the centripetal wave, demonstrating the effect of curvature on signal propagation.

All these observed behaviors were successfully captured by our ABFDF model. In particular, this model was used to describe the behavior of the two different signals (centripetal and centrifugal). This allowed us to compare the two signals and demonstrate the model's versatility.

In our model the potassium diffusion and degradation constants were homogenous across the biofilm and the potassium release function of all cells in the biofilm was also constant [ $\varepsilon$ in Eq. (11)]. Therefore, the spatial arrangement of the cells alone was enough to explain the varying velocity of the electrical wave fronts. Collectively these experimental and modeling results demonstrate how cell density and curvature can influence the propagation of an electrical signal in a biofilm.

It remains unclear what caused the centripetal waves that originated in the outer biofilm. They may be due to cross-talk with other neighboring biofilms, e.g., centrifugal waves that excite centripetal waves in neighboring biofilms. However, potassium has been shown to regulate and affect other key processes in B. subtilis cells [36,37], indicating a broader role for electrical signaling. There are also many other bacterial ion channels, besides Yug0 (a potassium channel), with a range of gating principles [13,38,39]. It is therefore logical to infer that electrical communication is likely to extend far beyond these initial applications. It is expected that signals are initiated by bacteria in response to a range of stimuli (e.g., a variety of stress responses) and that these signals illicit a broad range of different responses. As most cells are responsive to changes in local ion concentrations and potentials through the existence of voltage sensitive ion channels, it is probable that electrical signaling is more diverse than just intraspecies signaling.

Our ABFDF model can account for different cell behaviors and environmental conditions. It can therefore be adapted to model a wide variety of different systems and signals. With the expected expansion of the field of bacterial electrophysiology, such modeling techniques will become increasingly useful.

Future studies would profit from improved magnification in microscopy experiments (in the current study it was limited by the microfluidic cell used). This would allow the exact positioning of individual cells to be directly input into the agent-based model. In the current study the cell density was determined for coarser radial averages of the fluorescence images (Fig. 2), where individual bacterial positions could not be determined. A further improvement would be to study electrical wave propagation in 3D biofilms, since they are more commonly found in nature, although the interaction of electrical waves with boundaries of the biofilm that have widely varying curvatures and experience varying time delays is expected to markedly complicate matters. 
In conclusion, we have developed rigorous data analysis techniques and an ABFDF model to describe observed phenomena in the electrical signals of $B$. subtilis biofilms including the effect of spatial heterogeneity in bacterial cell placements and curvature of propagating wave fronts. More generally we have demonstrated the power of these methods in the emerging field of biofilm active excitable matter.

\section{ACKNOWLEDGMENTS}

The authors would like to thank the ESPRC for J.A.B.'s Ph.D. studentship and the MRC Research Grant No. EP/F062966/1 for support. They would also like to thank the Prindle group for useful discussions and the Gutiérrez group for their software and helpful discussions regarding its use.
[1] Y. Bashir, T. R. Betts, and K. Rajappan, Introduction to cardiac electrophysiology-a brief historical perspective, Cardiac Electrophysiology and Catheter Ablation, Oxford Specialist Handbooks in Cardiology (Oxford University Press, Oxford, 2010), pp. 2-6.

[2] T. A. Waigh, Some Critical Questions in Biological Physics: A Guided Tour around the Bugbears (IOP Publishing, Bristol, UK, 2017).

[3] M. Courtemanche, L. Glass, and J. P. Keener, Instabilities of a Propagating Pulse in a Ring of Excitable Media, Phys. Rev. Lett. 70, 2182 (1993).

[4] W.-J. Rappel, F. Fenton, and A. Karma, Spatiotemporal Control of Wave Instabilities in Cardiac Tissue, Phys. Rev. Lett. 83, 456 (1999).

[5] H.-C. Flemming, J. Wingender, U. Szewzyk, P. Steinberg, S. A. Rice, and S. Kjelleberg, Biofilms: An emergent form of bacterial life, Nat. Rev. Microbiol. 14, 563 (2016).

[6] H.-C. Flemming, T. R. Neu, and D. J. Wozniak, The EPS matrix: The 'house of biofilm cells', J. Bacteriol. 189, 7945 (2007).

[7] H. Vlamakis, Y. Chai, P. Beauregard, R. Losick, and R. Kolter, Sticking together: Building a biofilm the Bacillus subtilis way, Nat. Rev. Microbiol. 11, 157 (2013).

[8] N. Høiby et al., The clinical impact of bacterial biofilms, Int. J. Oral Sci. 3, 55 (2011).

[9] M. P. Schultz, J. A. Bendick, E. R. Holm, and W. M. Hertel, Economic impact of biofouling on a naval surface ship, Biofouling 27, 87 (2011).

[10] A. Kalziqi, D. Yanni, J. Thomas, S. L. Ng, S. Vivek, B. K. Hammer, and P. J. Yunker, Immotile Active Matter: Activity from Death and Reproduction, Phys. Rev. Lett. 120, 018101 (2018).

[11] T. Grafke, M. E. Cates, and E. Vanden-Eijnden, Spatiotemporal Self-Organization of Fluctuating Bacterial Colonies, Phys. Rev. Lett. 119, 188003 (2017).

[12] H. Jeckel et al., Learning the space-time phase diagram of bacterial swarm expansion, Proc. Natl. Acad. Sci. USA 116, 1489 (2019).

[13] P. Koprowski and A. Kubalski, Bacterial ion channels and their eukaryotic homologues, BioEssays 23, 1148 (2001).

[14] A. Prindle et al., Ion channels enable electrical communication in bacterial communities, Nature 527, 59 (2015).

[15] H. Beyenal and J. Babauta, Biofilms in Bioelectrochemical Systems (John Wiley \& Sons, Inc., New York, 2015).

[16] J. Liu et al., Coupling between distant biofilms and emergence of nutrient time-sharing, Science 356, 638 (2017).

[17] B. H. Kim and G. M. Gadd, Prokaryotic Metabolism and Physiology (Cambridge University Press, Cambridge, 2019).
[18] A. Verkhratsky and V. Parpura, History of Electrophysiology and the Patch Clamp BT-Patch-Clamp Methods and Protocols (Springer, New York, 2014).

[19] W. Gerstner and W. M. Kistler, Spiking Neuron Models: Single Neurons, Populations, Plasticity (Cambridge University Press, Cambridge, 2002).

[20] A. L. Hodgkin and A. F. Huxley, A quantitative description of membrane current and its application to conduction and excitation in nerve, J. Physiol. 117, 500 (1952).

[21] J. I. Vandenberg and S. G. Waxman, Hodgkin and Huxley and the basis for electrical signalling: A remarkable legacy still going strong, J. Physiol. 590, 2569 (2012).

[22] C. M. Glen, M. L. Kemp, and E. O. Voit, Agent-based modeling of morphogenetic systems: Advantages and challenges, PLoS Comput. Biol. 15, e1006577 (2019).

[23] Ş. Bora and S. Emek, Agent-based modeling and simulation of biological systems, Modeling and Computer Simulation (IntechOpen, London, 2019), Chap. 3.

[24] E. Ferrante, A. E. Turgut, M. Dorigo, and C. Huepe, ElasticityBased Mechanism for the Collective Motion of Self-Propelled Particles with Springlike Interactions: A Model System for Natural and Artificial Swarms, Phys. Rev. Lett. 111, 268302 (2013).

[25] C. A. Weber, C. Bock, and E. Frey, Defect-Mediated Phase Transitions in Active Soft Matter, Phys. Rev. Lett. 112, 168301 (2014).

[26] S. S. Branda, J. E. González-Pastor, S. Ben-Yehuda, R. Losick, and R. Kolter, Fruiting body formation by Bacillus subtilis, Proc. Natl. Acad. Sci. USA 98, 11621 (2001).

[27] M. Gutiérrez et al., A new improved and extended version of the multicell bacterial simulator gro, ACS Synth. Biol. 6, 1496 (2017).

[28] https://github.com/johannablee/Electrical-signalling-inbacterial-biofilms.

[29] G. Grimmett and D. Welsh, Probability: An Introduction (Oxford University Press, Oxford, 2014).

[30] M. S. Jafri and J. Keizer, On the roles of $\mathrm{Ca}^{2+}$ diffusion, $\mathrm{Ca}^{2+}$ buffers, and the endoplasmic reticulum in IP3-induced $\mathrm{Ca}^{2+}$ waves, Biophys. J. 69, 2139 (1995).

[31] J. Keener and J. Sneyd, Wave propagation in excitable systems, in Mathematical Physiology: I: Cellular Physiology, edited by J. Keener and J. Sneyd (Springer, New York, 2009), pp. 229-271.

[32] Z. Nagy-Ungvarai, J. Ungvarai, S. C. Müller, and B. Hess, The role of curvature and pulse width for transition to unstable wave fronts in the Belousov-Zhabotinsky reaction, J. Chem. Phys. 97, 1004 (1992).

[33] J. Keener and J. Sneyd, Calcium dynamics, Mathematical Physiology: I: Cellular Physiology (Springer, New York, 2010), p. 273. 
[34] P. S. Stewart, Diffusion in biofilms, J. Bacteriol. 185, 1485 (2003).

[35] E. Klavins, A language for modeling and programming cooperative control systems, in Proceedings of IEEE International Conference on Robotics and Automation, 2004 (IEEE, Piscataway, NJ, 2004), Vol. 4, pp. 3403-3410.

[36] M. E. Lundberg, E. C. Becker, and S. Choe, MstX and a putative potassium channel facilitate biofilm formation in Bacillus subtilis, PLoS One 8, e60993 (2013).
[37] J. B. Miller and D. E. Koshland, Sensory electrophysiology of bacteria: Relationship of the membrane potential to motility and chemotaxis in Bacillus subtilis, Proc. Natl. Acad. Sci. USA 74, 4752 (1977).

[38] I. R. Booth, M. D. Edwards, and S. Miller, Bacterial ion channels, Biochemistry 42, 10045 (2003).

[39] R. Koishi et al., A superfamily of voltage-gated sodium channels in bacteria, J. Biol. Chem. 279, 9532 (2004). 